\title{
Radix Table for Trigonometric Functions and Their Inverses to High Accuracy
}

1. Introduction.-By means of two algorithms, based upon the addition theorem for $\tan x$, and a radix table of $\arctan \left(m \cdot 10^{-n}\right), m=1(1) 9$, $n=1(1) 6$, to $20 \mathrm{D}$, one has the means of calculating $\tan x(x<\pi / 4)$ and arctan $x$ to at least $18 \mathrm{D}$ from a one-page table. The other trigonometric functions and their inverses follow easily. All division operations may be done upon an ordinary 10-bank desk calculator, so that no intermediate written work is necessary.

2. To obtain $\tan x$. - If $x<\frac{1}{4} \pi$, subtract from $x$ the largest tabular value of arctan $a_{1}$ that leaves a positive remainder $x_{1}$; from this remainder subtract the largest tabular value of $\arctan a_{2}$ that leaves a positive remainder $x_{2}$. Continue in this way until the remainder $x_{6}=x-\sum_{i=1}^{6} \arctan a_{i}$ is reached. The $a_{i}$ are all exact, each with a single significant digit, unless zero.

By evaluating in turn

$$
t_{6}=\tan x_{6} \doteq x_{6}, \quad t_{i-1}=\frac{t_{i}+a_{i}}{1-t_{i} a_{i}}, \quad i=6,5,4,3,2,1 ;
$$

one can obtain $t_{0}=t=\tan x$. An easier alternative is to evaluate in turn

$$
\begin{aligned}
a & =a_{1}+a_{2}, \quad b=a_{3}+a_{4}, \quad c=a_{5}+a_{6} ; \\
d & =1-a_{1} a_{2}, \quad e=1-a_{3} a_{4}, \quad f=1-a_{5} a_{6} ; \\
A_{1} & =a e+b d, \quad A_{2}=d e-a b, \\
B_{1} & =c+f x_{6}, \quad B_{2}=f-c x_{6} .
\end{aligned}
$$

Then $t=\tan x \doteq\left(A_{1} B_{2}+A_{2} B_{1}\right) /\left(A_{2} B_{2}-A_{1} B_{1}\right)$.

If $\frac{1}{4} \pi<x<\frac{1}{2} \pi$, evaluate $x^{\prime}=\frac{1}{2} \pi-x=1.57079632679489661923-x$; then $\tan x=1 / \tan x^{\prime}$ to as many significant figures as are known to 18 decimals in $x^{\prime}$.

Other functions are given by

$$
\begin{aligned}
& \sin x=t\left(1+t^{2}\right)^{-\frac{1}{2}}, \quad \cos x=\left(1+t^{2}\right)^{-\frac{1}{3}}, \quad \cot x=1 / t, \\
& \sec x=\left(1+t^{2}\right)^{\frac{1}{3}}, \quad \operatorname{cosec} x=\left(1+t^{2}\right)^{\frac{1}{3} / t} .
\end{aligned}
$$

3. To obtain $\arctan t$. - If $t<1$, evaluate in turn

$t_{1}=\left(t-a_{1}\right) /\left(1+a_{1} t\right)$ where $a_{1}$ is the first decimal digit of $t$,

$t_{2}=\left(t_{1}-a_{2}\right) /\left(1+a_{2} t_{1}\right)$ where $a_{2}$ is the second decimal digit of $t_{1}$, the first being zero, and so on, until $t_{6}$ is reached, using

$$
t_{i+1}^{\prime}=\left(t_{i}-a_{i+1}\right) /\left(1+a_{i+1} t_{i}\right) \text {. }
$$

Then $\arctan t \doteq \sum_{i=1}^{6} \arctan a_{i}+t_{6}$.

If desired, two successive stages can be combined, thus

$$
t_{i+2}=\frac{\left(1-a_{i+1} a_{i+2}\right) t_{i}-\left(a_{i+1}+a_{i+2}\right)}{1-a_{i+1} a_{i+2}+\left(a_{i+1}+a_{i+2}\right) t_{i}} .
$$


If $t>1, \quad \arctan t=\frac{1}{2} \pi-\arctan (1 / t)=1.57079632679489661923$ $-\arctan (1 / t)$. Other functions are given by

$$
\begin{gathered}
\operatorname{arccot} x=\frac{1}{2} \pi-\arctan x, \quad \arcsin x=\arctan \left(x\left(1-x^{2}\right)^{-\frac{1}{3}}\right), \\
\arccos x=\arctan \left(\left(1-x^{2}\right)^{\frac{1}{3}} / x\right), \quad \operatorname{arcsec} x=\arctan \left(x^{2}-1\right)^{\frac{1}{3},} \\
\operatorname{arccsc} x=\arctan \left(x^{2}-1\right)^{-\frac{1}{3}} .
\end{gathered}
$$

\begin{tabular}{|c|c|c|c|}
\hline $\begin{array}{l}a_{1} \\
.1 \\
.2 \\
.3 \\
.4 \\
.5 \\
.6 \\
.7 \\
.8 \\
.9\end{array}$ & 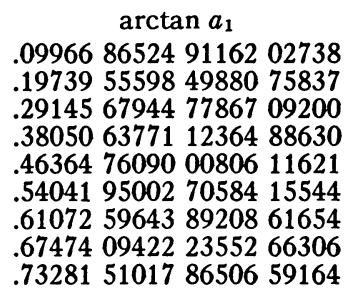 & $\begin{array}{r}a_{4} \\
.0001 \\
.0002 \\
.0003 \\
.0004 \\
.0005 \\
.0006 \\
.0007 \\
.0008 \\
.0009\end{array}$ & $\begin{array}{c}\arctan a_{4} \\
.00009999999966666667 \\
.00099999999733333340 \\
.00029999999100000049 \\
.00039999999866666871 \\
.00049999995833333958 \\
.00059999992800001555 \\
.0006999998 \quad 8566670028 \\
.00079999982933339887 \\
.000899999757000011810\end{array}$ \\
\hline $\begin{array}{l}a_{2} \\
.01 \\
.02 \\
.03 \\
.04 \\
.05 \\
.06 \\
.07 \\
.08 \\
.09\end{array}$ & 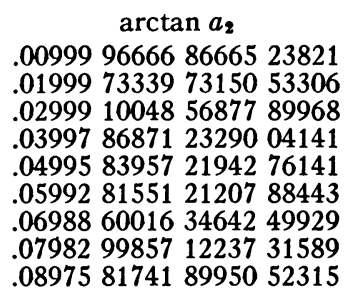 & $\begin{array}{c}a_{5} \\
.00001 \\
.00002 \\
.00003 \\
.00004 \\
.00005 \\
.00006 \\
.00007 \\
.00008 \\
.00009\end{array}$ & $\begin{array}{c}\arctan a_{5} \\
.00000999999999966667 \\
.00001999999999733333 \\
.00002999999999100000 \\
.00003999999997866667 \\
.00004999999995833333 \\
.00005999999992800000 \\
.00006999999988566667 \\
.00007999999982933333 \\
.00008999999975700000\end{array}$ \\
\hline $\begin{array}{l}a_{3} \\
.001 \\
.002 \\
.003 \\
.004 \\
.005 \\
.006 \\
.007 \\
.008 \\
.009\end{array}$ & 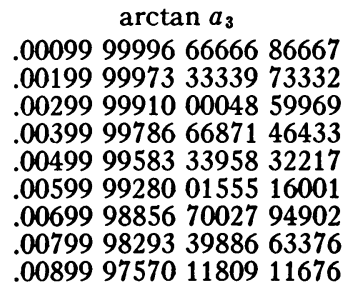 & $\begin{array}{c}a_{6} \\
.000001 \\
.000002 \\
.000003 \\
.000004 \\
.000005 \\
.000006 \\
.000007 \\
.000008 \\
.000009\end{array}$ & $\begin{array}{c}\arctan a_{6} \\
.000000999999999999967 \\
.000001999999999999733 \\
.00000299999999999100 \\
.00000399999999997867 \\
.00000499999999995833 \\
.00000599999999992800 \\
.00000699999999988567 \\
.00000799999999982933 \\
.00000899999999975700\end{array}$ \\
\hline
\end{tabular}

TABLE OF ARCTAN $a_{i}$.

\section{Examples.}

A. To obtain $\tan x$, for $x=.56548667764616278292$ :

Here $\arctan .6$ is seen to be the largest value of $\arctan a_{1}$ such that $x-\arctan a_{1}$ is positive. From

$x-\arctan .6=x_{1}=.02506717737557862748, a_{2}$ is seen to be .02 . From From

$x_{1}-\arctan .02=x_{2}=.00506984340242809442, a_{3}$ is seen to be .005 .

$x_{2}-\arctan .005=x_{3}=.00006988506846977225, a_{4}$ is seen to be 0 , $x_{3}=x_{4}$, and $a_{5}$ is seen to be .00006 . From

$x_{4}-\arctan .00006=x_{5}=.00000988506854177225, a_{6}$ is seen to be .000009 . Finally

$x_{5}-\arctan .000009=x_{6}=.00000088506854201525$.

$a=a_{1}+a_{2}=.62, b=a_{3}+a_{4}=.0050, c=a_{5}+a_{6}=.000069$,

$d=1-a_{1} a_{2}=.988, e=1-a_{3} a_{4}=1, f=1-a_{5} a_{6}=.99999999946$;

$A_{1}=a e+b d=.62494, A_{2}=d e-a b=.9849$, 
$B_{1}=c+f x_{6}=.00006988506854153731$,

$B_{2}=f-c x_{6}=.99999999939893027060$.

$A_{1} B_{2}=.62493999962436748331, A_{2} B_{1}=.00006882980400656010$, $A_{2} B_{2}=.98489999940800642351, A_{1} B_{1}=.00004367397473434833$, $A_{1} B_{2}+A_{2} B_{1}=.62500882942837404341$, $A_{2} B_{2}-A_{1} B_{1}=.98485632543327207518$, $t=\tan x \doteq \frac{A_{1} B_{2}+A_{2} B_{1}}{A_{2} B_{2}-A_{1} B_{1}}=.634619297544148 \quad 10040$,

which is correct to about 3 units in the 19th decimal place.

B. To obtain $\arctan t$, for $t=.59139835139947109817$ :

Since $a_{1}=.5$, we have

$$
\begin{gathered}
t_{1}=\left(t-a_{1}\right) /\left(1+a_{1} t\right)=\frac{.09139835139947109817}{1.295699175699355491}, \\
\text { or } t_{1}=.07053979281147617252, a_{2}=.07 ; \\
t_{2}=\left(t_{1}-a_{2}\right) /\left(1+a_{2} t_{1}\right)=\frac{.000539792811176 \quad 17252}{1.0049377854967823321}, \\
\text { or } t_{2}=.00053714052647481117, a_{3}=0 ; \\
t_{3}=t_{2}, a_{4}=.0005 ; \\
t_{4}=\left(t_{3}-a_{4}\right) /\left(1+a_{4} t_{3}\right)=\frac{.00003714052647481117}{1.00000026857026324}, \\
\text { or } t_{4}=.00003714051649997288, a_{5}=.00003 ; \\
t_{5}=\left(t_{4}-a_{5}\right) /\left(1+a_{5} t_{4}\right)=\frac{.00000714051649997288}{1.000000001 \quad 1142155}, \\
\text { or } t_{5}=.00000714051649201681, a_{6}=.000007 ; \\
t_{6}=\left(t_{5}-a_{6}\right) /\left(1+a_{6} t_{5}\right)=\frac{.00000014051649201681}{1.000000000499836}, \\
\text { or } t_{6}=.00000014051649200979 .
\end{gathered}
$$

$\arctan t=\arctan .5+\arctan .07+\arctan .0005+\arctan .00003$

$$
+\arctan .000007+.00000014051649200979 \text {, }
$$$$
\text { or } \arctan t=.53407075111026485054 \text {, }
$$

which happens to be correct to 20 decimals.

NBSCL

H. E. SAlZER

\section{RECENT MATHEMATICAL TABLES}

834[E].-L. Prandtl \& F. Vandrey, "Fliessgesetze normal-zäher Stoffe im Rohr. Ein Beitrag zur Rheologie," Zeit. angew. Math. Mech., v. 30, 1950, p. $169-174$.

This article gives two tables of functions having to do with viscous flow. The function $\phi(a)$ is defined by

$$
\phi(a)=8 \sum_{n=1}^{\infty} n(2 n+1) a^{2 n-2} /(2 n+2) !
$$

